\title{
EFEITO DO ESPAÇAMENTO NA PRODUTIVIDADE DE BIOMASSA DE TAXI-BRANCO (Sclerolobium paniculatum Vogel)
}

\author{
Alberto William Viana de CASTRO', João Tomé de FARIAS NETO², Emanuel \\ da Silva CAVALCANTE ${ }^{3}$
}

RESUMO - Visando determinar o efeito de diferentes espaçamentos sóbre o comportamento silvicultural do taxi-branco (Sclerolobium paniculatum Vogel) foi instalado experimento no Campo Experimental do Cerrado pertencente ao Centro de Pesquisa Agroflorestal do Amapá - Embrapa Amapá, no Estado do Amapá, Brasil. Foram empregadas quatro repetiçôes em experimento delineado em blocos casualisados e sete tratamentos correspondentes aos espaçamentos: $2,0 \mathrm{~m} \times 1,0 \mathrm{~m}$; $1,5 \mathrm{~m} \times 1,5 \mathrm{~m} ; 3,0 \mathrm{~m} \times 1,0 \mathrm{~m} ; 2,0 \mathrm{~m} \times 2,0 \mathrm{~m} ; 2,5 \mathrm{~m} \times 2,0 \mathrm{~m} ; 3,0 \mathrm{~m} \times 2,0 \mathrm{~m} ; 3,0 \mathrm{~m} \times 2,5 \mathrm{~m}$. As análises foram feitas à idade de sete anos. Os resultados evidenciaram diferenças não significativas para os parâmetros altura, DAP, sobrevivência, e número de fustes, Quanto ao parâmetro biomassa foi detectado somente dois grupos de médias estatisticamente diferentes. A menor produção foi obtida no espaçamento $3,0 \mathrm{mx} 2,0 \mathrm{~m}$ enquanto que a maior produção foi verificada no espaçamento $2,0 \mathrm{mx} 1,0 \mathrm{~m}$ com $1.355 \mathrm{~kg}$ semelhante estatisticamente das produções obtidas nos espaçamentos $1,5 \mathrm{mxl}, 5 \mathrm{~m}$ com $1.351 \mathrm{~kg}$ e $3,0 \mathrm{~m} \times 1,0 \mathrm{~m}$ com $1.091 \mathrm{~kg}$ havendo portanto concordância plena entre produção de biomassa e densidade de plantio. De um modo geral, nota-se que o espaçamento silvicultural mais indicado para o taxi-branco nas condições de cerrado amapaense é o $3,0 \mathrm{mx1}, 0 \mathrm{~m}$, visto que além de utilizar menor nủmero de plantas e portanto deve apresentar menor custo de produção, atingiu produtividade de biomassa semelhante estatisticamente aos menores espaçamentos.

Palavras-chaves: taxi-branco, espaçamento, biomassa, altura, DAP.

\section{Effects of Crop Spacing on Biomass Productivity of Taxi-Branco (Sclerolobium paniculatum Vogel).}

ABSTRACT - A crop density trail was carried out at the Cerrado Experiment Station, Embrapa Amapá, Amapá, Brazil, to evaluate the effects of density on biomass yield of taxi-branco (Sclerolobium paniculatum Vogel). The trial tested seven spacings $(2 \times 1 \mathrm{~m}-5000$ trees/ha, $1.5 \times$ $1.5 \mathrm{~m}-4444 \mathrm{t} / \mathrm{ha}, 3 \times 1 \mathrm{~m}-3333 \mathrm{t} / \mathrm{ha}, 2 \times 2 \mathrm{~m}-2500 \mathrm{t} / \mathrm{ha}, 3 \times 2 \mathrm{~m}-1666 \mathrm{t} / \mathrm{ha}, 3 \times 2.5 \mathrm{~m}-1333$ tha) in a randomized block design with four replications; growth and yield were measured in the seventh year, Height, diameter (DBH), survival and stem number showed no differences among treatments. There were two groups of treatments that differed in terms of biomass: the lowest yield was found at $3 \times 2 \mathrm{~m}$, while the highest yields were $1.355 \mathrm{t}$ at $2 \times 1 \mathrm{~m}, 1.351 \mathrm{t}$ at $1.5 \times 1.5 \mathrm{~m}$, and $1.091 \mathrm{t}$ at $3 \times 1 \mathrm{~m}$, so that there was good agreement between density and yield. Overall the best spacing for the Amapá cerrado conditions were $3 \times 1 \mathrm{~m}$, which uses fewer plants, has a lower cost, and yields similar to the higher density treatments.

Key words: height, diameter, biomass yield, density.

\section{INTRODUÇÃO}

As possibilidades de utilização para fins econômicos das áreas de cerrado, no Estado do Amapá fazem das espécies florestais uma real opção. Estudo realizado nesse ecossistema, revelou excelente comportamento silvicultural do taxibranco, expresso pelo incremento médio anual em altura de $2,2 \mathrm{~m} / \mathrm{ano}$, diâmetro a

Eng. Ftal., M.Sc. EMBRAPA/Centro de Pesquisa Agroflorestal da Amazônia Oriental (CPATU), Caixa Postal 48, CEP 66095-100, Belém, PA.

2 Eng. Agro,, Dr., EMBRAPA/Centro de Pesquisa Agroflorestal do Amapá (CPAF-AP). Caixa Postal 10, CEP 68902-280, Macapá, AP.

3 Eng. Agro., M.Sc., EMBRAPA/Centro de Pesquisa Agroflorestal do Amapá (CPAF-AP). Caixa Postal 10, CEP 68902-280, Macapá, AP 
altura do peito (DAP) de $2,9 \mathrm{~cm} / \mathrm{ano}$, sobrevivência de $90 \%$ e árvores com forma e vigor excelentes (Castro et al., 1990).

Trata-se de uma espécie arbórea nativa de terra firme da região amazônica, pertencente a família das leguminosae, subfamilia caesalpinoideae. $\mathrm{O}$ interesse pelo taxi-branco deve-se a produção de madeira de boa qualidade para produção de carvão, aliada a capacidade de associação com bactérias fixadoras de $\mathrm{N}$ atmosférico, rápido crescimento acompanhado de elevada produção e desrama de folhas, possibilitando uma rápida formação de "litter", mesmo em solos álicos e de baixa fertilidade. Tais características conferem potencial para sua utilização em áreas alteradas pela ação antrópica (Dias et al., 1995).

A escolha do espaçamento de plantio na maioria dos planejamentos florestais, tem sido fundamentada simplesmente no uso final da madeira, negligenciando-se outros fatores envolvidos de ordem ecológicos/ silviculturais de suma importância. $\mathrm{O}$ espaçamento tem uma série de implicações do ponto de vista silvicultural, tecnológico e econômico. Diferenças significativas na taxa de sobrevivência, nas taxas de crescimento das plantas, na qualidade da madeira e idade de corte têm sido detectadas, bem como nas práticas de exploração e manejo florestal, e, consequentemente, nos custos de produção. De fato, Kageyama et al. (1987) encontrou cinco diferentes tendências de resposta ao aumento do espaçamento em diferentes grupos de espécies arbóreas nativas, desde positiva em crescimento com o aumento do espaçamento até negativa.
Em condições brasileiras, a maioria dos plantios comerciais tem sido implantada graças aos estudos desenvolvidos visando à produção de madeira para celulose e /ou chapas de fibras. Considerando-se a diversidade de comportamento das espécies florestais e as diferentes qualidades de madeira exigidas para cada uso, acredita-se que o espaçamento ideal para celulose não seja o mesmo indicado para produção de lenha, carvão ou madeira para serraria (Simões et al., 1981), havendo, desse modo a necessidade de se definir o espaçamento adequado para a condução das florestas comerciais com a finalidade de produzir árvores com boas características para os mais diversos fins. De acordo com o exposto, este trabalho teve como finalidade determinar o efeito de diferentes espaçamentos sobre o comportamento silvicultural do taxi-branco, considerando seu potencial silvicultural como excelente produtora de matéria prima para a produção de carvão vegetal.

\section{MATERIAL E MÉTODOS}

As mudas utilizadas na instalação do experimento, foram provenientes da mistura de sementes de 21 árvores selecionadas na Floresta Nacional do Tapajós (FLONA) no município de Belterra no Estado do Pará.

$\mathrm{O}$ experimento foi instalado em 1989, no Campo Experimental do Centro de Pesquisa Agroflorestal do Amapá (CPAF-AP), localizado a $0^{\circ} 22$, de latitude $\mathrm{N}$ e $51^{\circ} 04$, de longitude $\mathrm{W}$ e $50 \mathrm{~m}$ de altitude, aproximadamente a $45 \mathrm{~km}$ da cidade de Macapá. A área apresenta topografia plana, coberta com vegetação de cerrado, clima tipo Ami 
segundo a classificação de Köppen, temperatura média anual de $27^{\circ} \mathrm{C}$, umidade média relativa do ar de $82 \%$ e precipitação média de $2300 \mathrm{~mm}$ (Boletim, 1990). O solo é do tipo Latossolo Amarelo, textura média e de baixa fertilidade. Foi utilizada uma única adubação, realizada na ocasião do plantio, empregando-se $100 \mathrm{~g} /$ cova de NPK (15-30-15) + 5g de bórax + $5 \mathrm{~g}$ de sulfato de zinco.

O delineamento experimental foi em blocos casualisados, com os tratamentos representados por sete espaçamentos (Tab. 1), distribuidos em quatro repetições.

Cinco parâmetros foram avaliados aos sete anos de idade, sendo obtidas as medidas de altura, DAP, número de fustes e sobrevivência. A biomassa (BMS)' é um parâmetro estimado pela seguinte expressão: $\mathrm{BMS}=-3.64+$ $0.593 \times \mathrm{CSA}$, onde CSA = somatório dos diâmetros ao quadrado dos troncos existentes na mesma árvore a uma altura de $1,30 \mathrm{~m}$ e foi incluída como parâmetro medidor da produção de madeira.

A análise de variância, foi realizada segundo os procedimentos usuais de um delineamento de blocos casualisados. Em árvores bifurcadas ou trifurcadas, o DAP e a altura foram obtidos através da média dos fustes. $\mathrm{O}$ parâmetro sobrevivência, para efeito da análise de variância, foi transformado em arc. sen. $x$.

\section{RESULTADOS E DISCUSSÃO}

Os valores e significâncias dos quadrados médios (teste F) detectados na análise de variância para os parâmetros altura da planta, DAP, sobrevivência, número de fustes $\mathrm{e}$ biomassa, são apresentados na tabela 2 . Conforme pode ser observado, os coeficientes ฟde variação experimental estão dentro dos limites aceitáveis da experimentação agrícola, pois variaram de $7,4 \%$ (sobrevivência) a $15,7 \%$ (biomassa). Nota-se que, ao nível dos tratamentos (espaçamentos), foi constatada diferença altamente significativa somente para o parâmetro biomassa, refletindo um comportamento diferencial para esse parâmetro nos diferentes espaçamentos empregados.

Na tabela 3, encontram-se as estimativas médias de altura, DAP e

Tabela 1. Tratamentos empregados, número de plantas avaliadas, área/árvore e densidade.

\begin{tabular}{lcccc}
\hline Tratamento & $\begin{array}{c}\text { Espaçamento } \\
(\mathrm{m} \times \mathrm{m})\end{array}$ & $\begin{array}{c}\mathrm{N} . \text { o de plantas } \\
\text { por parcelas }\end{array}$ & $\begin{array}{c}\text { Área/árvore } \\
\left(\mathrm{m}^{2)}\right.\end{array}$ & $\begin{array}{c}\text { Densidade } \\
\text { (árvores/ha) }\end{array}$ \\
\hline 1 & $2,0 \times 1,0$ & 72 & 2,0 & 5.000 \\
2 & $1,5 \times 1,5$ & 64 & 2,25 & 4.444 \\
3 & $3,0 \times 1,0$ & 48 & 3,0 & 3.333 \\
4 & $2,0 \times 2,0$ & 36 & 4,0 & 2.500 \\
5 & $2,5 \times 2,0$ & 30 & 5,0 & 2,000 \\
6 & $3,0 \times 2,0$ & 24 & 6,0 & 1.667 \\
7 & $3,0 \times 2,5$ & 20 & 7,5 & 1.333 \\
\hline
\end{tabular}

Informação pessoal prestada pelo Dr, Milton Kanashiro (Embrapa Amazônia Oriental) 
Tabela 2. Quadrados médios obtidos para cinco parâmetros em taxi-branco aos sete anos de idade. Macapá-AP, 1996.

\begin{tabular}{lcccccc}
\hline Fonte de & GL & \multicolumn{3}{c}{ Quadrados Médios } \\
\cline { 3 - 7 } Variação & & Altura & DAP & Sobrevivência & N. de Fustes & Biomassa \\
\hline Blocos & 03 & 0,29 & 2,04 & 53,9 & 0,14 & 519724,86 \\
Tratamentos & 06 & $0,43 \mathrm{~ns}$ & $0,53 \mathrm{~ns}$ & $32,24 \mathrm{~ns}$ & $0,16 \mathrm{~ns}$ & $366246,72 * *$ \\
Residuo & 18 & 0,18 & 0,25 & 32,7 & 0,06 & 22558,16 \\
\hline Média Geral & & 4,2 & 4,99 & 77,8 & 1,8 & 956,1 \\
CV (\%) & 10,1 & 10 & 7,4 & 12,6 & 15,7 \\
Unidade & $\mathrm{m}$ & $\mathrm{cm}$ & $\%$. & & $\mathrm{n} / \mathrm{parcela}$ & $\mathrm{kg} / \mathrm{parcela}$ \\
\hline
\end{tabular}

** : significativo a $1 \%$ de probabilidade. ns: nào significativo.

sobrevivência. Verifica-se que esses parâmetros não sofreram influência dos diferentes espaçamentos, sugerindo que aos sete anos a influência da concorrência por luz e/ou nutrientes do solo, nos diversos espaçamentos, ainda não foi atingida. Tal fato também foi verificado por Barros (1962) com duas espécies arbóreas nativas sob diferentes espaçamentos aos cinco anos de idade. Entretanto, nota-se que os maiores incrementos médios em altura e DAP ocorreram em espaçamentos maiores. Scolforo (1990), observou que nos sítios mais produtivos ocorre uma maior variabilidade dos diâmetros que nos sitios menos produtivos, como é o caso do cerrado amapaense. Nesses sítios, espera-se uma menor amplitude entre a menor e maior classe diamétrica, o que traduz um ritmo de crescimento menos intenso das árvores.

$\mathrm{O}$ número de plantas mortas em espaçamentos menores foi praticamente igual ao verificado nos espaçamentos maiores, sugerindo que o autodesbaste das plantas nos menores espaçamentos deve ocorrer em idades mais avançadas.

Quanto ao parâmetro biomassa, diferenças significativas ao nivel de $1 \%$ de probabilidade foram detectadas. As maiores produções de biomassa foram verificadas nos espaçamentos menores, onde se encontra maior número de árvores por unidade de área. Assim, o espaçamento $2,0 \mathrm{~m} \times 1,0 \mathrm{~m}$ alcançou produtividade média de 1355 $\mathrm{kg} /$ parcela seguido dos espaçamentos $1,5 \mathrm{mx} 1,5 \mathrm{~m}$ com $1.351 \mathrm{~kg} /$ parcela $\mathrm{e}$ $3,0 \mathrm{mxl}, 0 \mathrm{~m}$ com $1091 \mathrm{~kg} /$ parcela. Estes resultados concordam com as observações de Fishwich (1976), que afirma serem as diferenças entre a produção volumétrica de povoamentos de espaçamentos pequenos e de povoamentos de espaçamentos mais amplos, nos primeiros anos, causados pelo fato de que as árvores mais espaçadas e de rápido crescimento, não estão utilizando todo o espaçamento disponivel para crescer. Por isso, os povoamentos mais densos, atingem o volume do sítio mais rapidamente.

Considerando-se a biomassa produzida, nota-se que o espaçamento silvicultural mais indicado é o $3,0 \mathrm{~m} \times 1,0 \mathrm{~m}$, visto que utiliza menor número de árvores e portanto deve 
Tabela 3. Estimativas de médias de altura, DAP, sobrevivência, biomassa e número de fustes em taxi-branco aos sete anos de idade sob diferentes espaçamentos. Macapá-AP, 1996.

\begin{tabular}{lccccc}
\hline Espaçamentos & $\begin{array}{c}\text { Altura } \\
(\mathrm{m})\end{array}$ & $\begin{array}{c}\text { DAP } \\
(\mathrm{cm})\end{array}$ & $\begin{array}{c}\text { Sobrevivência } \\
(\%)\end{array}$ & $\begin{array}{c}\text { Biomassa } \\
(\mathrm{kg} / \text { parcela })\end{array}$ & $\begin{array}{c}\text { N. } \text { Fustes } \\
(\mathrm{n} . \% \text { parcela })\end{array}$ \\
\hline $3,0 \times 2,5$ & 4,44 & 5,41 & 94 & $726 \mathrm{~b}$ & 2,0 \\
$3,0 \times 2,0$ & 4,36 & 5,51 & 96 & $719 \mathrm{~b}$ & 1,9 \\
$2,5 \times 2,0$ & 3,83 & 4,66 & 93 & $725 \mathrm{~b}$ & 2,0 \\
$2,0 \times 2,0$ & 3,75 & 4,70 & 97 & $725 \mathrm{~b}$ & 1,7 \\
$3,0 \times 1,0$ & 4,67 & 5,16 & 96 & $1091 \mathrm{a}$ & 1,7 \\
$1,5 \times 1,5$ & 4,15 & 4,81 & 93 & $1351 \mathrm{a}$ & 1,8 \\
$2,0 \times 1,0$ & 4,23 & 4,71 & 94 & $1355 \mathrm{a}$ & 1,6 \\
\hline
\end{tabular}

1) As médias seguidas pela mesma letra nāo diferem entre si ao nível de $5 \%$ de probabilidade.

apresentar menor custo de produção. Além disso, atingiu estatisticamente igual produtividade em relação às maiores densidades de plantio. Entretanto, sugerese realizar uma análise econômica em relação aos custos de implantação, custos de exploração entre outros, em termos de decisão comercial.

\section{CONCLUSÕES}

Os resultados obtidos neste trabalho, permitem as seguintes conclusões:

1.Não foi detectado efeito significativo da densidade de plantio sobre a altura, DAP, sobrevivência e número de fustes aos sete anos de idade, indicando que esses parâmetros são independentes da densidade de plantio.

2. A produção de biomassa foi significativamente influenciada pela densidade de plantio, sendo as maiores produções de biomassa obtidas nos menores espaçamentos.

3. O espaçamento $3,0 \mathrm{mxl}, 0 \mathrm{~m}$ mostrou-se ser o mais indicado silviculturalmente, para as condições do cerrado amapaense, visando a produção de matéria prima para a produção de carvão vegetal com o taxi-branco, visto que apresentou a produção de biomassa estatisticamente igual às menores densidades de plantio.

\section{Bibliografia citada}

Boletim Agrometeorológico. 1990. Macapá: EMBRAPA-UEPAE de Macapá, 1990. 55p.

Barros, D.P. 1962. Competição de espécies e espaçamentos. Silvicultura em Sào Paulo, São Paulo, v. 1, n.2, p. 93-106,

Castro, A.W.V. de ; Yared, J.A.G; Alves, R.N.B.; Silva, L.S.; Meirelles, S.M.L.B. 1990. Comportamento silvicultural de Sclerolobium paniculatum (Taxi-branco) no cerrado amapaense. Macapá, AP: EMBRAPA-UEPAE de Macapá, 4p. (EMBRAPA-UEPAE de Macapá. Comunicado Técnico, 07).

Dias, L.E.; Brienza Junior, S.; Pereira, C.A.1995. Taxi branco (Sclerolobium paniculatum Vogel): Uma leguminosa arbórea nativa da Amazônia com potencial para recuperação de áreas degradadas. In: Kanashiro, M.; Parrota, J.A. (eds). Manejo e reabilitação de àreas degradadas e florestas secundárias na amazônia. Paris, France: UNESCO, p. 148-153,

Fishwich, R.W. 1976. Estudos de espaçamento e desbastes em plantações brasileiras. 
Brasil Florestal, Rio de Janeiro, v. 7, n. 26, p. 13-23,

Kageyama, P.Y.; Bila, A.; Luima, J. 1987

Critérios de escolha de espécies para utilização em pequenas áreas de reflorestamento, Brasilia: IBDF, .18p.

Scolforo, J.R.S. 1990. Sistema integrado para predição e análíse presente e futura do crescimento e produção, com otimização de remuneração de capitais para Pinus caribaea var. hondurensis. Curitiba, PR:UFPR, 290P. Tese Doutorado.

Simōes, J.W; Brandi, R.M.; Leite, N.B.; Balloni, E.A. 1981. Formaçâo, manejo e exploração de florestas com espécies de rápido crescimento. Brasilia, DF: IBDF, 131p. 